\title{
Gamification at Scraim
}

\author{
Diogo Silva ${ }^{1(凶)}$, António Coelho ${ }^{1}$, César Duarte ${ }^{2}$, and Pedro Castro Henriques ${ }^{2}$ \\ 1 DEI/FEUP, INESC TEC, Porto, Portugal \\ diogoemsilva@gmail.com, acoelho@fe.up.pt \\ 2 Strongstep, Porto, Portugal \\ \{cesar.duarte, pedroch\}@strongstep.pt
}

\begin{abstract}
Software developers usually work as a team and in order to improve the team organization and performance several companies use agile methodologies. The efficiency of this methodologies can be improved by the use of project management tools. Several software developers are difficult to motivate on the usage of these tools, as they often claim being disruptive to their workflow.

This paper presents the use of gamification to incite software developers to use this kind of tools with the necessary regularity. The main focus of this work is on the tasks completion and the regular use of the project management tool, while insuring the respect of the values proposed by the agile manifesto to software development.

The gamification process is described in detail, followed by an application the project management software SCRAIM.
\end{abstract}

Keywords: Gamification - Game design · Software management tools • Agile manifesto

\section{Introduction}

The use of project management tools based on Agile methodologies for software development brings benefits to the project members. While the project manager can distribute the work efficiently, the development team knows what to do in every moment.

Towards a correct use of these tools, the development team is often forced to disrupt their workflow to fill in the required information on these tools. Although this is not a negative factor by itself it may affect the efficacy of these tools in such manner that the use may not be the desirable. In this research project we use SCRAIM, an online project and process management platform that has the goal to promote better organizational practices.

To engage software development teams on the use of the project management tools we applied gamification, in order to incorporate game elements and game design techniques to motivate them.

The problem identified is how to create a gamification strategy that engages software developers to make a better use of these tools. 
To do this we considered the following research questions:

- What's the most suitable combination of game elements?

- How to mitigate the risks associated to gamification?

- How to ensure the values of the manifesto for agile software development?

Following these research questions we defined the following goals:

- Develop a survey to help understanding the motivating/blockers aspects of the use of project management tools.

- Design a gamification approach for SCRAIM

- Develop a prototype to integrate this gamification approach in SCRAIM.

- Test and validate the developed strategy.

\section{State of Art and Related Work}

This section presents the manifesto for the agile software development and the concept of gamification and also some related work.

Software development undergoes several steps since the requirements identification to the product release. It is necessary to have a plan, although usually this plan may be adjusted if and when necessary. Agile software development is an adaptive process that considers [1]:

- Individuals and interactions over processes and tools

- Working software over comprehensive documentation

- Customer collaboration over contract negotiation

- Responding to change over following a plan

Gamification is defined as the use of game elements and game design techniques in non-game contexts [2]. Different combination of game elements may offer different user experiences. One particular common combination is $\mathrm{PBL}$ (points, badges and leaderboards) [3].

Gamification has a strong component of psychology, particularly related to motivation. Intrinsic activities are the ones where the user does something in order to fulfil personal goals while extrinsic activities uses external motivators as money [4]. Extrinsic motivators can be a strong motivational tool but may bring some risks [3].

Although it may bring several benefits, gamification has some critics that is necessary to be aware. First the rewards may be a problem if misused or overused. We should be aware that rewards usually are extrinsic motivators so they must be used as a mean to a end (usually intrinsic motivators as personal goals) [2]. Secondly gamification may be used to explore users (exploitation), not only by replacing tangible rewards by intangible ones with less value but it may also incite an unfavorable behavior [5]. At least some game elements uses in gamification strategies may cause law issues. So it is necessary to be aware about laws that may be applied as privacy laws or gambling laws. 
Gamification in the workplace has some aspects that are necessary to be considered as the organization hierarchical structure and the motivation mechanics already implemented. Money as a motivational factor must be used with care, always to promote the professionalism without disrespecting the people and promote quality over quantity. [4]

In terms of related work, StackOverflow is a very famous programming assistive platform. The mechanic is simple, the user just asks questions and answers questions from other users. It may also classify answers if they are good or not. Through this actions, the user earns reputation points. This points may be used to unlock new features as moderation tools. The user may also earn some badges if certain conditions are met.

Another example of gamification is RedCritter Tracker. It's an agile project management tool that uses gamification as differential factor. In this tool the users earn points by doing tasks. This points may be used to buy items in the virtual shop and the users may also earn badges if certain condition are met.

As we can see, there are some game elements that are used often such as points (reputation points or virtual currency), badges and leaderboards, known as the PBL triad.

SCRAIM is the project and process management tool in which this research is focused. It follows an agile methodology and has several features as project planning, work distribution, project overview showing statistics as burdown chart and risks management.

\section{Gamification Process}

In order to create the gamification process to incorporate it on SCRAIM, we followed the Werbach's "six D" step process:

- Define business objectives. They must be clear and objective and not a mean to an end. The identified objectives were: engagement of the correct use of SCRAIM by software developers, improve its efficiency and provide mutual aid.

- Delineate target behaviours and define metrics to evaluate them. The identified behaviours were: fill task details and check for the main fields, complete tasks, collaboration between project elements, improve workplace culture through the use of surprise element, consult leaderboards, promote mutual aid and access gamification options.

- Describe the players. Identify who are the players that will play and try to understand their point of view. To do this several personnel were developed in order to understand the different kinds of users (according to Kim's model $\left.(2010)^{1}\right)$.

- Devise activity cycles. Be aware that there is different game phases that need to be considered. The cycles identified were the daily challenge system where a

\footnotetext{
${ }^{1}$ http://amyjokim.com/blog/2012/09/19/social-engagement-whos-playing-how-dothey-like-to-engage/.
} 
new challenge is presented every day to user when he access SCRAIM for the first time, the help request system where a user can answer help requests and make new ones and a competition for best team using SCRAIM in a sprint.

- Don't forget the fun. It's a fundamental aspect of the games. The fun factor is present in daily challenges, help requests, competition of good practices and the mascot (this element serves as a mean for the user to access gamification options outside the default workflow of SCRAIM).

- Deploy the appropriate tools. This step must be the logic result of all the previous steps.

In order to find the motivating and blockers aspects of this kind of tools, interviews were done to nine students of Masters in Informatics of FEUP. These happened before the beginning of the conception of the strategy and it served as a starter point. During the interviews users were asked about what motivates them for use this kind or tools or not. Most of the users said that this tool is great for work distribution and planning. That is great for the users to organise themselves and in a work team. About the blocking aspects, several users pointed the interface as the main blocking aspect.

As a result of several iterations of this process, the following game elements where integrated on SCRAIM:

Daily challenge system

Users propose challenges to each other and one of them is chosen each day challenge. That offers a playful space in the tool while promoting surprise to entice a regular use by the users.

Helping system

This system allows users to help each other. Besides facilitating the problem resolution, the answers can be consulted later in such manner that helps similar problems in the future.

Good practices of using SCRAIM

In order to promote a better use of the tool, we developed a competition between existing projects. Each task has a score depending on how well it is filled. The collection of task's scores grants an overall score to the project.

Mascot

Given the enterprise context of the tool, the proposed components were developed in such manner that are not intrusive to the user (an example is the use of pop-ups to view challenges and help requests). On the other hand, a mascot was created that, through notifications, works as a connection between the tool and the gamification components while keeping the user's autonomy.

While developing the gamification process, a second series of interviews were performed in order to validate the strategy. These interviews were done to five students of Masters in Informatics of FEUP. The main aspects of the strategy were discussed towards finding what would be possible to improve. In the interviews, the users were presented with several mock-ups and they are asked to give their opinion about them. The main conclusions were: 
- It is interesting to use feedback mechanisms

- Simple and non intrusive game elements must be used in order not to disrupt the workflow

- Gamification options must be outside of the main workflow of the tool

- Avoid the use of negative connotations on user evaluations (e.g. leaderboards).

\section{Evaluation and Results}

In order to validate the developed strategy, the test methodology consisted in two parts: an interview using think-aloud protocol and a quiz.

The interviews were conducted to collaborators of Strongstep because they already knew the product so they could measure the impact of gamification in SCRAIM. Besides these collaborators other people were included from different areas such as informatics, management, design and economy so they could act differently regarding their experience and skill.

In the first part, several tasks were presented to the interviewed for them to execute and to express everything that they feel. This follows a think-aloud protocol that allows to capture and evaluate the user experience. The tasks asked consisted in the user to interact with the developed system in order to:

- Examine the gamification mascot.

- Open the daily challenge, answer it and give some feedback.

- Open the notifications center.

- Open a notification and check its content.

- Open a help request and answer it.

- Create a challenge.

- Create a help request.

- Check the gamification area for challenge and help request's statistics and hall of fame.

- Check task's gamification bar (it is a progress bar that shows if the task has the main fields properly filled).

- Check the project view for the members contribution table.

The second part of the interview was a survey with the purpose to evaluate the user experience in parameters such as simplicity and necessity. This survey was also a complement to the think-aloud protocol since it would capture details that users did't express. The survey asked the user to grade the components of the developed strategy (Daily challenge system, helping system, "Good pratices of using SCRAIM" and notifications) using parameters as "it is useful?", "it is fun?", "it is necessary?" and "Would you use it regularly?" in classification such "Strongly disagree", "Disagree", "Neither disagree or agree", "Agree" and "Strongly agree". Besides, it was asked if the use of gamification encourages a better use of SCRAIM.

The results showed that in general the developed strategy had a positive impact in SCRAIM. The number of collaborators interviews was six. This may be a small number of results but they are valuable to a future development of 
this work. All of the users knew SCRAIM and the concept of gamification and they agree that it is a potential way to improve and increase the use of the tool.

Regarding the challenge system, it showed up simple and intuitive in a general way, where the surprise factor attracts the user to use the tool at least daily. The users suggest improvement in the creation of the challenge regarding mainly the way that the correct answer is chosen.

Regarding the help system, the users identified the necessity of this system on SCRAIM. It was interesting the fact that half of the users don't agree that this system was fun. This can be explained by the existence of several perspectives of fun [4]. The work environment also may contribute to this review since helping a team member is part of the job.

Regarding the presence of gamification in the tasks, the users considered as a good way to promote the good pratices of using SCRAIM mainly because it is fun and provides a quick feedback.

Regarding the presence of the mascot with notifications in SCRAIM, it was considered as a bridge that connects the developed solution with the scope of the rest of the tool. Some users were confused with notification at first. One possible solution would provide a greater information about notification and their purpose.

\section{Conclusions}

Considering the results, it is possible to conclude that the developed prototype had a positive impact on the tool. The strong points identified were:

- The use of simple and intuitive elements. That way, the users don't need additional time to learn how to use the tool.

- Use of non-intrusive elements. This gives the user the choice of using the gamification component without disrupting his workflow in the tool.

- Promotion of the interaction between users though mutual aid.

- Use of the surprise element as a way to attract the user to use the tool at least daily.

- Promotion of the correct way to use the tool.

In this paper several research questions were answered in order to solve the identified problem. At this point we can present some conclusions:

- What's the most suitable combination of game elements? At this context the chosen combination of game elements must be simple and intuitive in such manner that allows a simple interaction with a user without being intrusive, promotes the user autonomy and respects the enterprise environment.

- How to avoid the hazards associated to gamification? Defining simple and fair metrics, design a simple feedback mechanics, avoids negative connotation and promotes interaction between users.

- How to ensure the proposed values of the manifesto to agile software development? In order to respect these values, the strategy was designed in such manner that the good use of the tool is a mean to the improvement of users relationships. 
Acknowledgements. The research leading to these results has received funding from the European Union's Horizon 2020 - The EU Framework Programme for Research and Innovation 2014-2020, under grant agreement No. 687676.

\section{References}

1. Manifesto for Agile Software Development. http://www.agilemanifesto.org/

2. Deterding, S., Dixon, D., Khaled, R., Nacke, L.: From game design elements to gamefulness. In: Proceedings of the 15th International Academic MindTrek Conference on Envisioning Future Media Environments - MindTrek 2011 (2011)

3. Werbach, K., Hunter, D.: For the Win: How Game Thinking Can Revolutionize Your Business. Wharton Digital Press, New York (2012)

4. Dale, S.: Gamification: Making Work Fun, or Making Fun of Work? Business Information Review. SAGE Publications Ltd, New York (2014)

5. Bogost, I.: Persuasive games: exploitationware. Gamasutra (2011). http://goo.gl/ jK1VR 